\title{
Novel Cassane and Cleistanthane Diterpenes from Myrospermum frutescens: Absolute Stereochemistry of the Cassane Diterpenes Series.
}

Daniel Torres-Mendoza, ${ }^{\dagger}$ Luis David Ureña-González, ${ }^{\dagger}$ Eduardo Ortega-Barría, ${ }^{\dagger}$ Phyllis D. Coley, ${ }^{\Uparrow \S}$ Thomas A. Kursar, ${ }^{\Phi \S}$ Todd L. Capson, ${ }^{\S}$ Kerry McPhail, ${ }^{\infty}$ Luis Cubilla-Rios* ${ }^{\dagger}$

Laboratory of Tropical Bioorganic Chemistry, Faculty of Natural, Exact Sciences and Technology, Apdo. 0824-10835, University of Panama, Panama City, Republic of Panama; Institute for Advance Scientific Investigation And Technology Services, National Secretariat of Science and Technology, Clayton, Ancon, Republic of Panama; Department of Biology, University of Utah, Salt Lake City, UT; Smithsonian Tropical Research Institute, Balboa, Ancon Republic of Panama and College of Pharmacy, Oregon State University, Corvallis, OR, 97331.

* To whom correspondence should be addressed. Tel: (507) 681 5371, Fax: (507) 264 4450.

E-mail: 1ucr@ancon.up.ac.pa

\section{Table of Contents}

S1. $\quad{ }^{1} \mathrm{H}$ NMR Spectrum for Chagreslactone (3)

S2. $\quad{ }^{13}$ C NMR Spectrum for Chagreslactone (3)

S3. HSQC Spectrum for Chagreslactone (3)

S4. HMBC Spectrum for Chagreslactone (3)

S5. COSY Spectrum for Chagreslactone (3)

S6. $\quad{ }^{1} \mathrm{H}$ NMR Spectrum for Chagresnone (4)

S7. $\quad{ }^{13} \mathrm{C}$ NMR Spectrum for Chagresnone (4)

S8. HSQC Spectrum for Chagresnone (4)

S9. HMBC Spectrum for Chagresnone (4)

S10. COSY Spectrum for Chagresnone (4)

${ }^{\dagger}$ University of Panama.

"Institute for Advance Scientific Investigation and High Technology Services.

"University of Utah.

${ }^{\S}$ Smithsonian Tropical Research Institute.

${ }^{a}$ Oregon State University. 


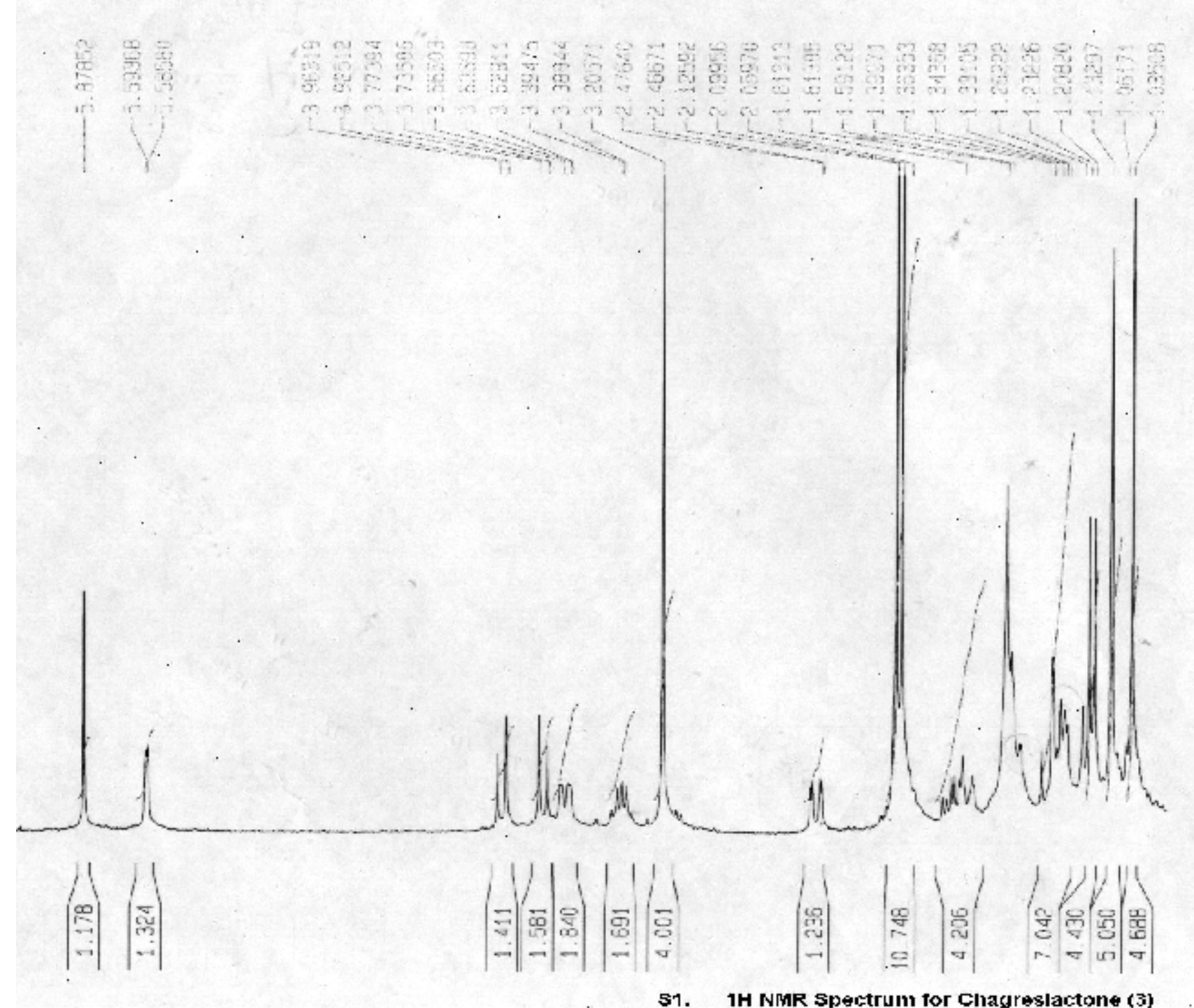

Current Data Parsmaters VAME MO1-183-0C1 ExpNo 20
1

-2 - Acquisition Paraleters

Cate_ 20030215

The

80.35

INSITHUM

FAOEIID $5 \mathrm{~mm}$ nie $13 .-1$

FULPROG 2970

To

SOL VFN'

NS

as

5172.939 H2

$\begin{array}{lr}0.094190 \mathrm{He} \\ 40 & 5.7084560 \mathrm{Hcc}\end{array}$

RC

Dh 21 2000 y=e

12.00 usec

D1 $1.00000000=0$

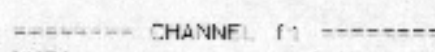
NUC1 $1 \mathrm{H}$
P1 10.00 utsec

$200.13 .05 \cdot 4$

FE - Processing parameters

$\begin{array}{cc}\mathrm{S}= & 32753 \\ \mathrm{~S}= & 300.1300021 \mathrm{MH}\end{array}$

nDN $\quad$ EM

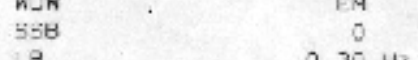

$\begin{array}{ccc}38 & 0.30 & 16\end{array}$

10 Na plut parameter

10 Nap plot parameters
Cx $2000 \mathrm{~cm}$

$\begin{array}{ll}\mathrm{CX} & 20.00 \mathrm{~cm} \\ \mathrm{CY} & 12.90 \mathrm{~cm} \\ \text { FIP } & \mathrm{E} .054 \mathrm{pFm}\end{array}$

F1 2420.39 Hz

$\begin{array}{rr}\text { Fip } & 0.072 \mathrm{pH} \\ \mathrm{F} 2 & 261.75 \mathrm{~Hz}\end{array}$

$\begin{array}{ll}\text { F2 } & 261.75 \mathrm{~Hz} \\ \text { PPMCM } & 0.35 .496 ? \mathrm{nnm} / \mathrm{Cm}\end{array}$

HZCM $107.93191 \mathrm{~Hz} / \mathrm{Cm}$

51. 1H NMR Spetrum for Chagreslactone (3) 



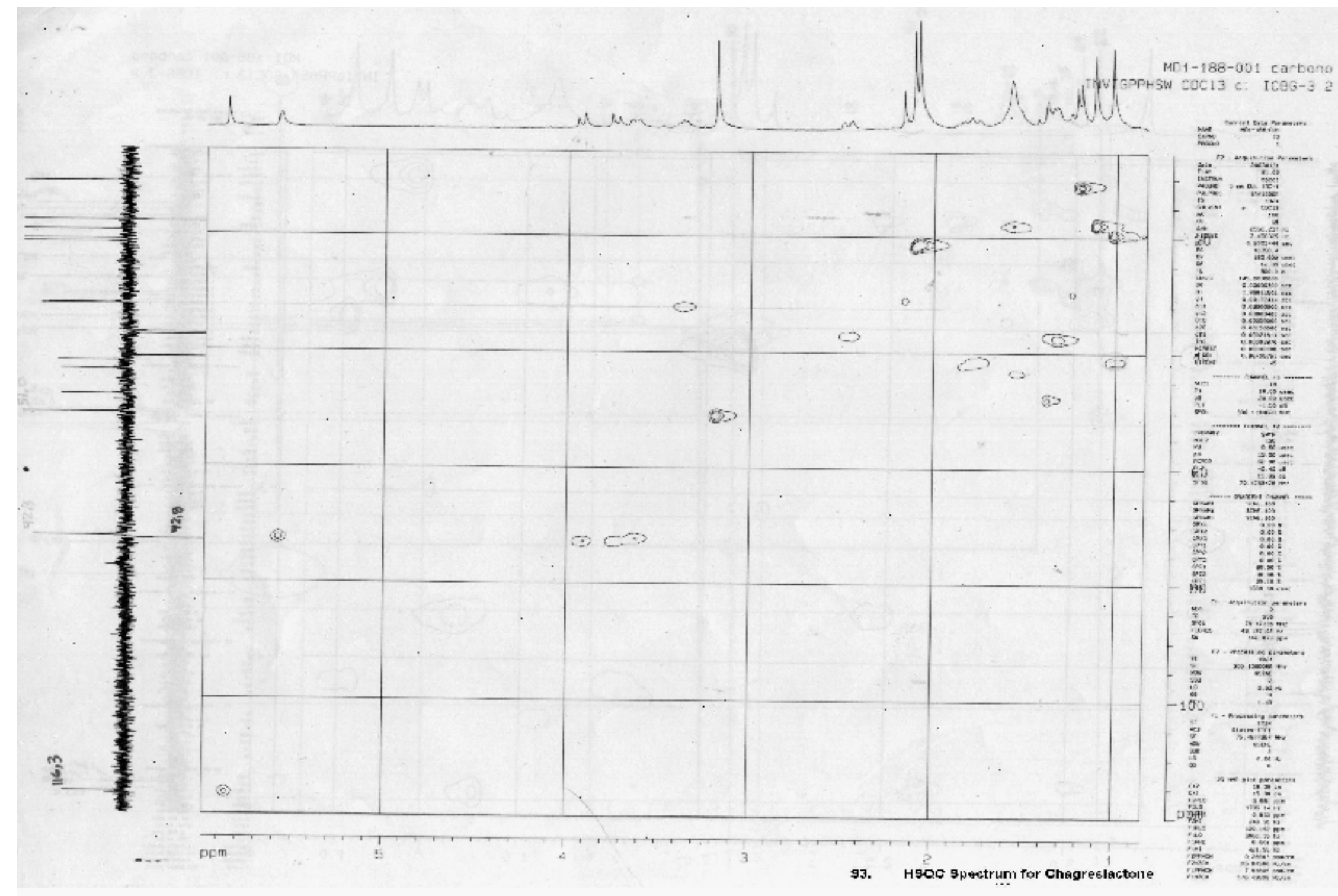




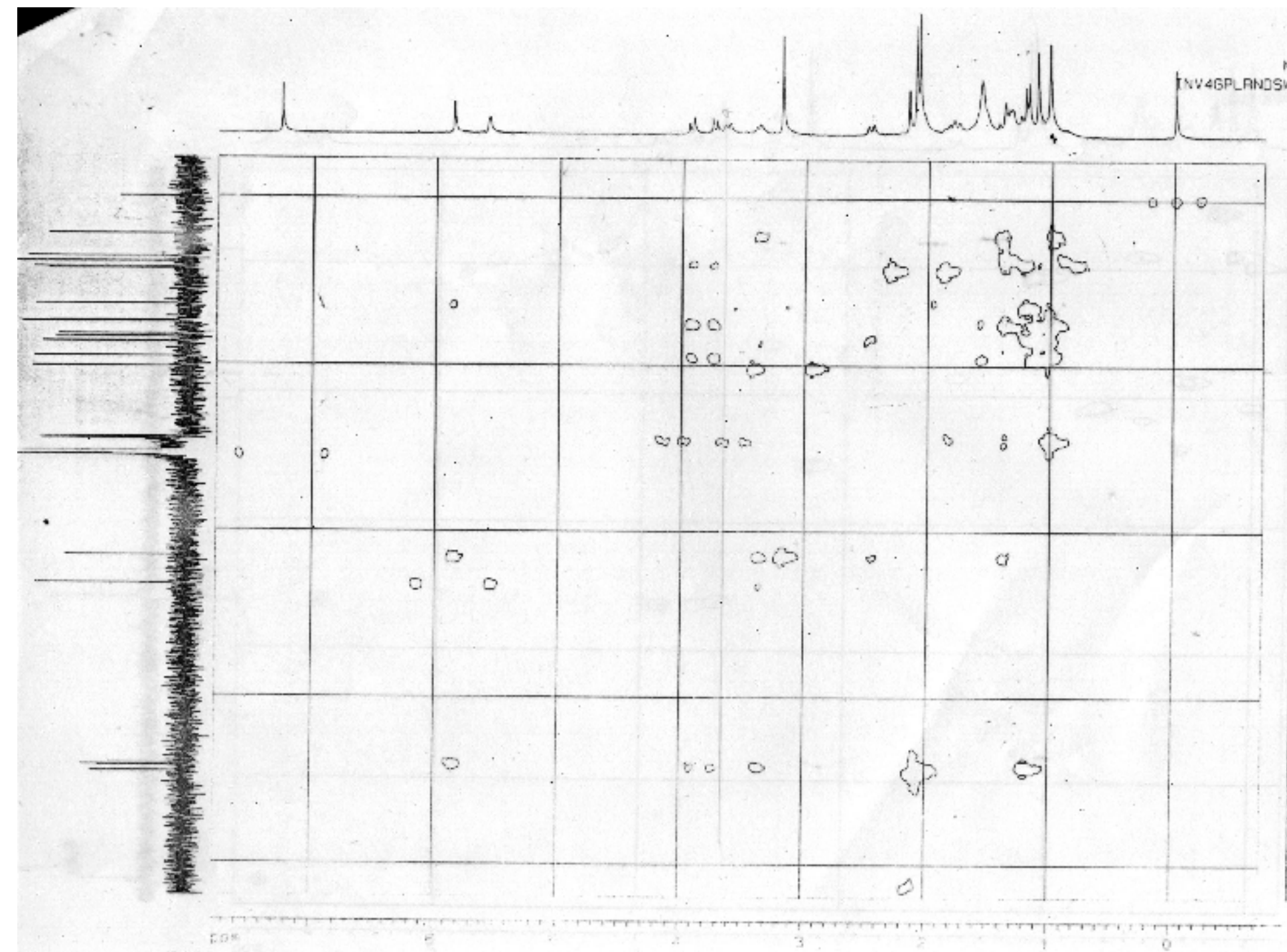

MD 1-18E-00: carbonc Cac $13 \mathrm{c}$ : Icas-3

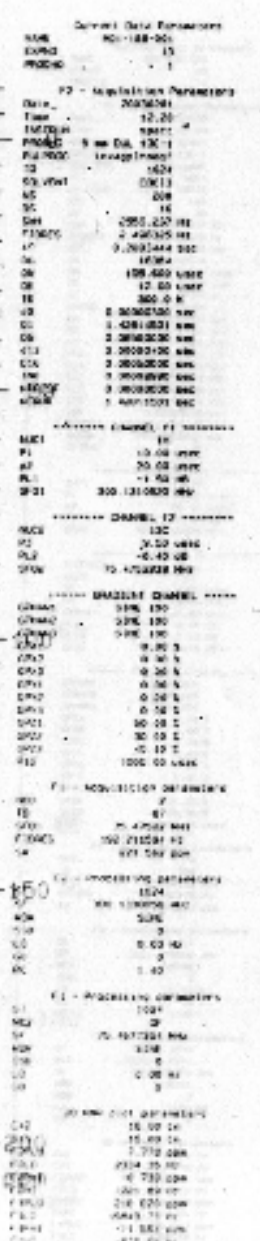

54. HMBe spectrum for thagreslactone 

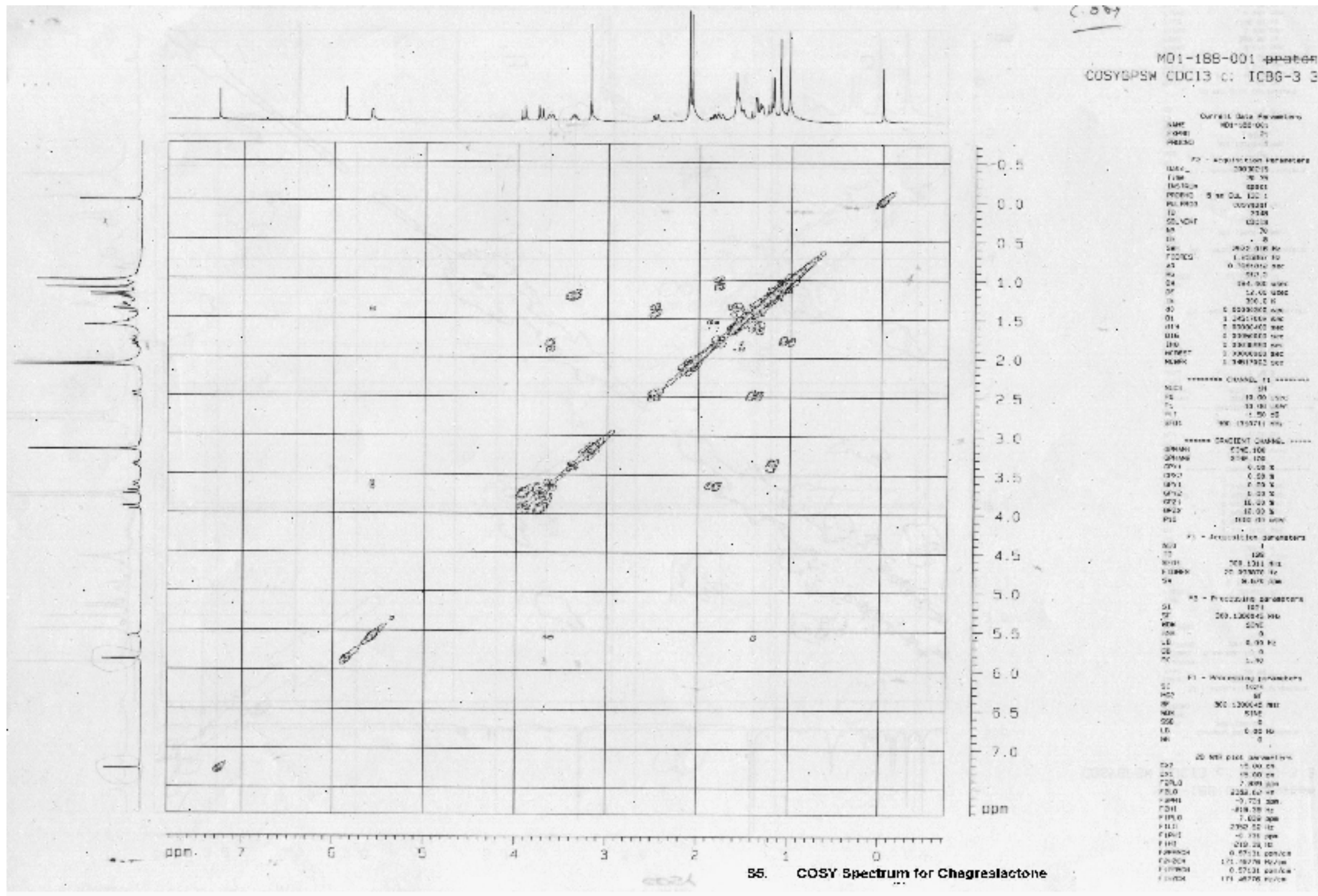


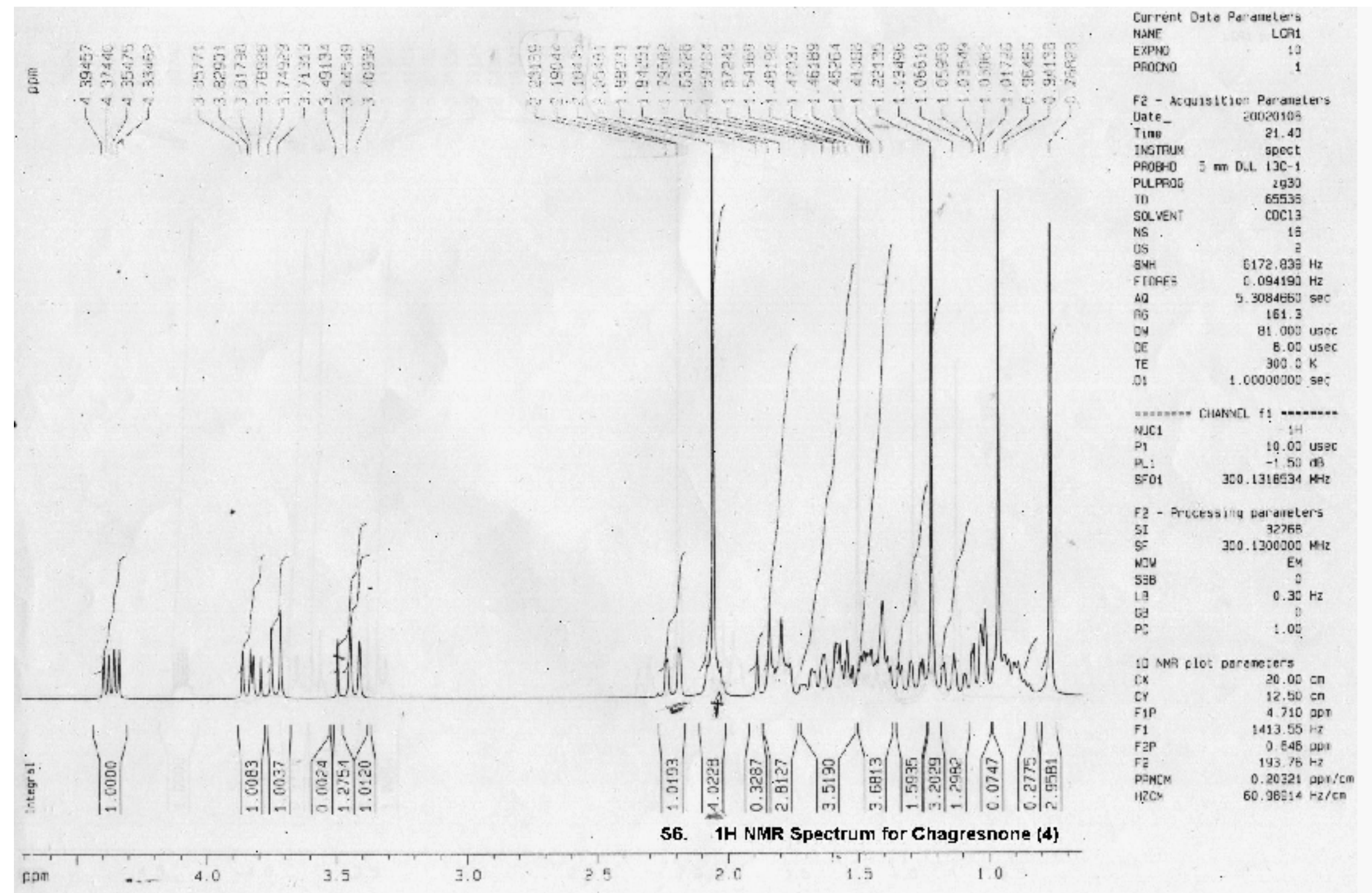




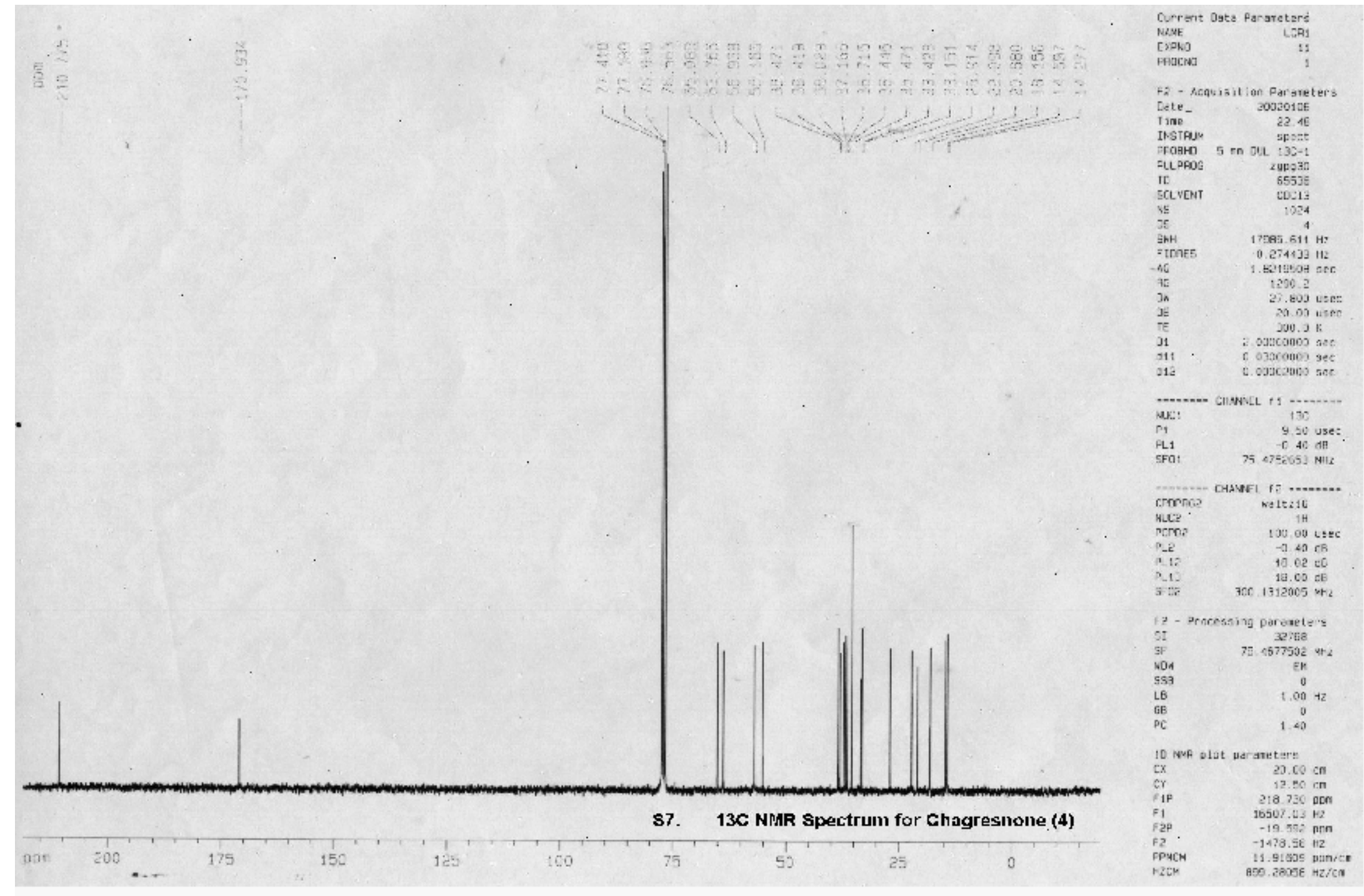




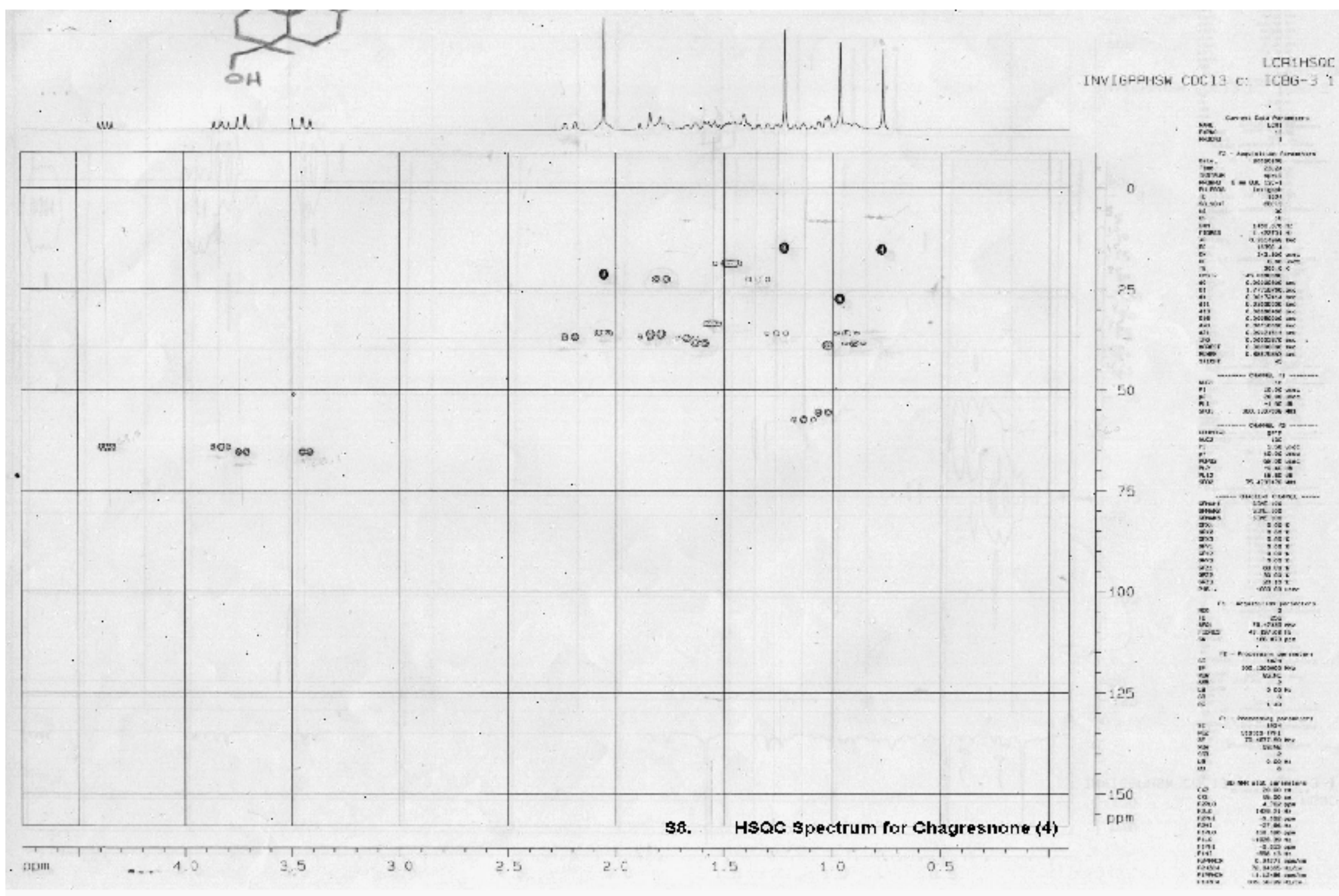




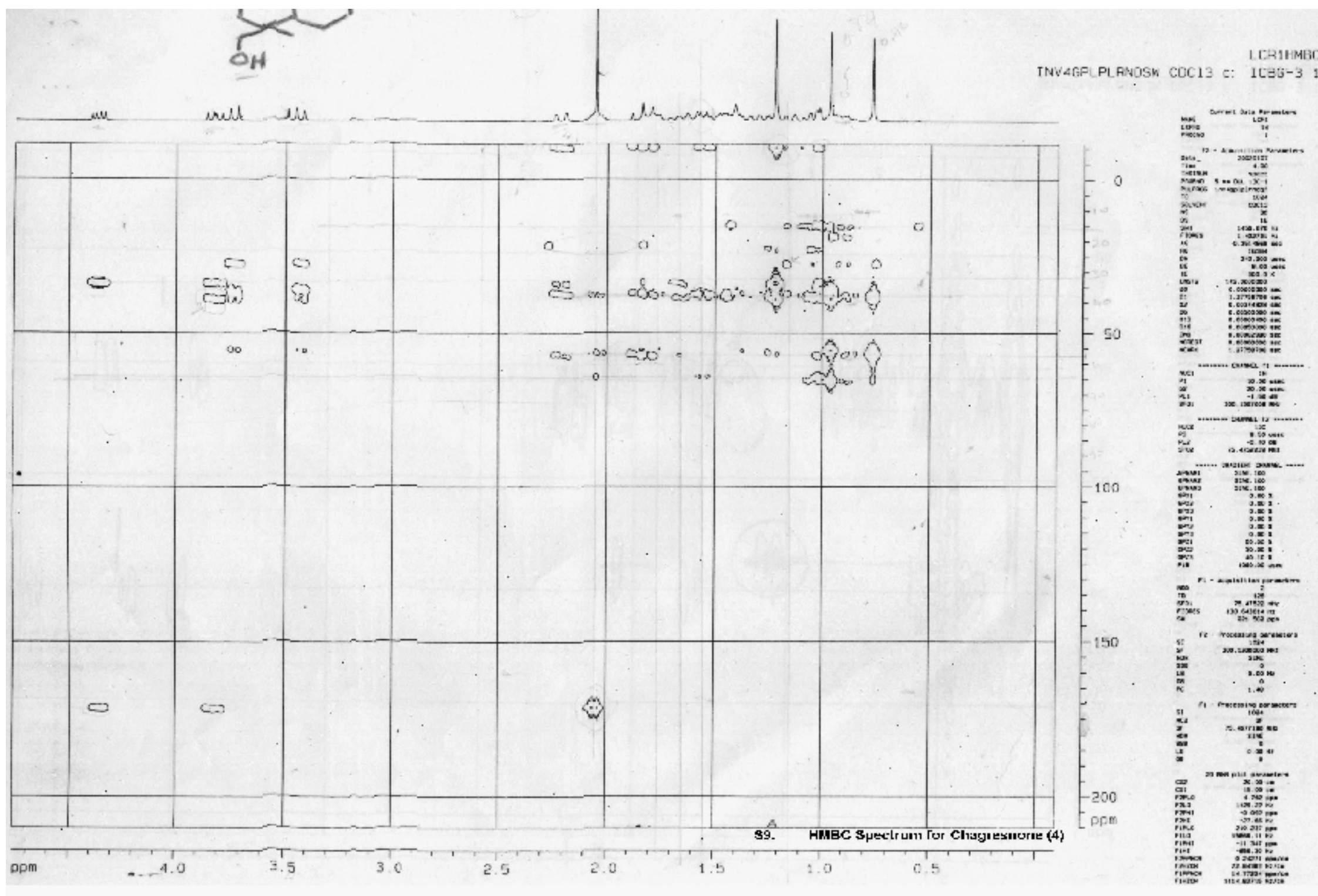




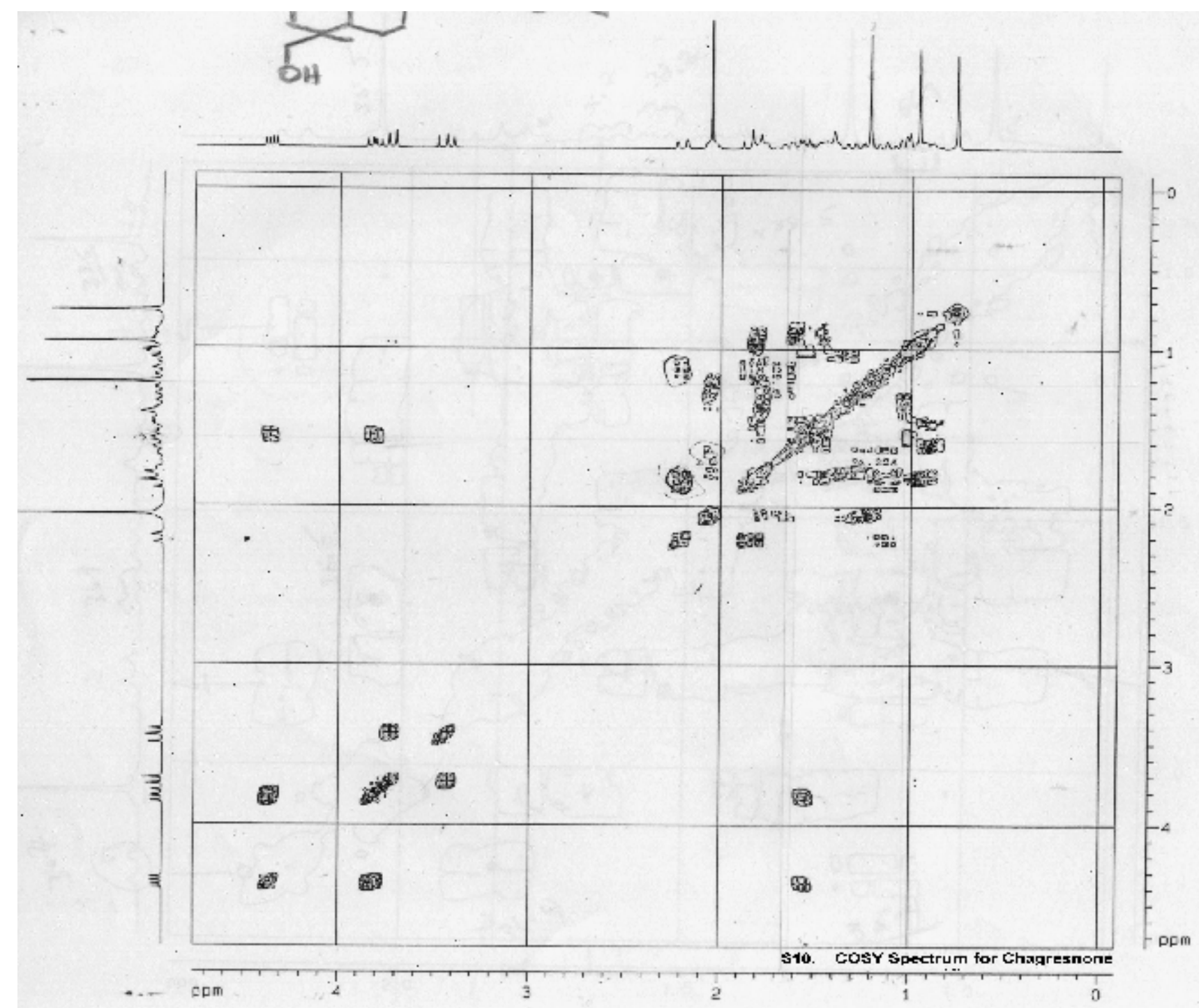

CDSYASEN COC13 LCR1EHAC COSY

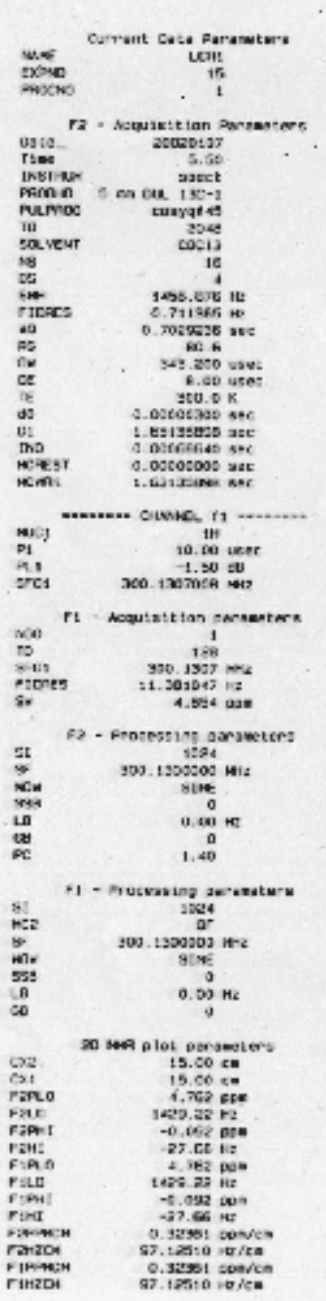

